\section{Wire to the Heart: A Unique Case of Psychosis}

\section{Thomas J Blount, Varun Monga* Kellie Nelson and Ashish Sharma}

Department of Psychiatry, University of Nebraska Medicine Center, Omaha, Nebraska, USA

\begin{abstract}
Non-suicidal self-injury is a common occurrence in the population of individuals who suffer from schizophrenia, schizoaffective disorder, bipolar disorder, and other major psychiatric disorders. Many case reports have detailed self-inflicted injury to the eyes, genitals, or face in patients with major psychiatric disorders. We report an unusual occurrence of non-suicidal self-injury which consisted of the insertion of 7 copper wires into the chest cavity of an incarcerated 44 year old man suffering from schizoaffective disorder, which resulted in perforation of the cardiac muscle. The patient was diagnosed with schizoaffective disorder at age 18, and has attempted trials of several psychotropic medications. He was switched to clozapine approximately six months before the incident, and states that he declined his medications for several days before the self-mutilation. This patient denied suicidal ideation, and reports that his self-injury was a result of command hallucinations. We believe this to be a unique presentation of self-injurious behavior in a man with schizoaffective disorder.
\end{abstract}

\section{Introduction}

Psychosis is a phenomenon that manifests in manners which are intensely subjective and present differently from patient to patient [1]. Schizophrenia and schizoaffective disorder are two common psychotic disorders associated with suicidal behaviors and also with non-suicidal self-harm gestures. Schizoaffective disorder is diagnosed according to criteria set forth in DSM-V. The diagnosis requires that the patient exhibit concurrently features of a major mood episode and schizophrenic criteria (hallucinations, delusions, etc.) for an uninterrupted period of illness. Additionally, the patient must exhibit during the lifetime of their illness at least 2 weeks of psychotic symptoms in the absence of mood symptoms [2]. Often, self-injurious acts are prompted by auditory command hallucinations that urge the patient to injure himself or herself to serve some delusional gain [3]. The following case presents what we believe to be a unique example of self-mutilating behavior in response to auditory hallucinations.

*Corresponding author: Varun Monga, Department of Psychiatry, University of Nebraska Medicine Center, Omaha, Nebraska, USA, Tel: +1 4025171315; Fax: +1 4025526247; E-mail: monga.varun81@gmail.com

Citation: Blount TJ, Monga V, Nelson K, Sharma A (2015) Wire to the Heart: A Unique Case of Psychosis. J Clin Stud Med Case Rep 2: 016.

Received: April 10, 2015; Accepted: June 05, 2015; Published: June 24, 2015

\section{Case Report}

We present the case of a 44-year-old Caucasian male who is currently incarcerated, and was seen by our psychiatric consult team while he was admitted to the hospital primarily for medical treatment. The patient was diagnosed with schizoaffective disorder at age 18, and his prominent psychotic symptoms include command auditory hallucinations and delusions. He has also been diagnosed with obsessive compulsive disorder and generalized anxiety disorder. Prior to his incarceration 4 years ago, the patient regularly abused methamphetamine starting at the age of 18 and continuing for about 10 years. He also abused alcohol and smoked 1 pack of cigarettes per day up until his arrest and incarceration. He has not used any drugs, alcohol, or nicotine products since being in prison.

The patient's disease course included auditory command hallucinations that constantly urged him "to do bad things." Prior to his incarceration he had been hospitalized several times for psychiatric reasons and overtime, his condition became refractory to treatment after trials of several antipsychotic medications. Since age 18 , he has attempted suicide several times, each time the attempt has been by slitting his wrists. Of note, he does not have any history of non-suicidal deliberate self-mutilation. Due to the treatment refractoriness of his disorder, his psychiatry team in prison started him on clozapine six months prior to this incident. His dose was titrated to $300 \mathrm{mg}$ per day in split doses. Since starting clozapine, he reports that his disease has been well controlled, besides persistent auditory hallucinations which he hears coming from within his own head. However, he clarifies that the voices are not heard nearly as often or as loudly as they were before he started on clozapine.

Approximately 3 weeks before he was admitted to our care, the patient began to feel that his medication regimen was making him feel cognitively blunted. In response to these effects, he admits several consecutive days during which he declined his medications. In turn, he began to experience a dramatic increase in the severity and urgency of his auditory hallucinations and paranoia.

In response to his command auditory hallucinations, the patient cut a 5-inch segment of his television coaxial cable and peeled the insulation from the cord. Over the next several days, he slowly pushed 7 copper wires into his chest around the location of his heart. He was rushed to the emergency department when he was found with several pulsating wires protruding from his chest. Upon his presentation to the emergency department, he was agitated and visibly responding to internal stimuli, though apparently euthymic. He was initially unable to explain the wires and was immediately transferred to the cardiology floor for further evaluation and management. The cardiology team first took a chest $x$-ray, which revealed 7 separate 5 -inch segments of copper wires that were bent in various directions in the his chest cavity. This $\mathrm{x}$-ray is presented as figure 1 .

When questioned later about the incident, the patient adamantly reported that this was not a suicide attempt. In the hospital, he stated "I know it looks like a suicide attempt, but it definitely wasn't". Malingering was also ruled out, as there was no obvious opportunity for secondary gain in this case. The patient reports that the act was a 


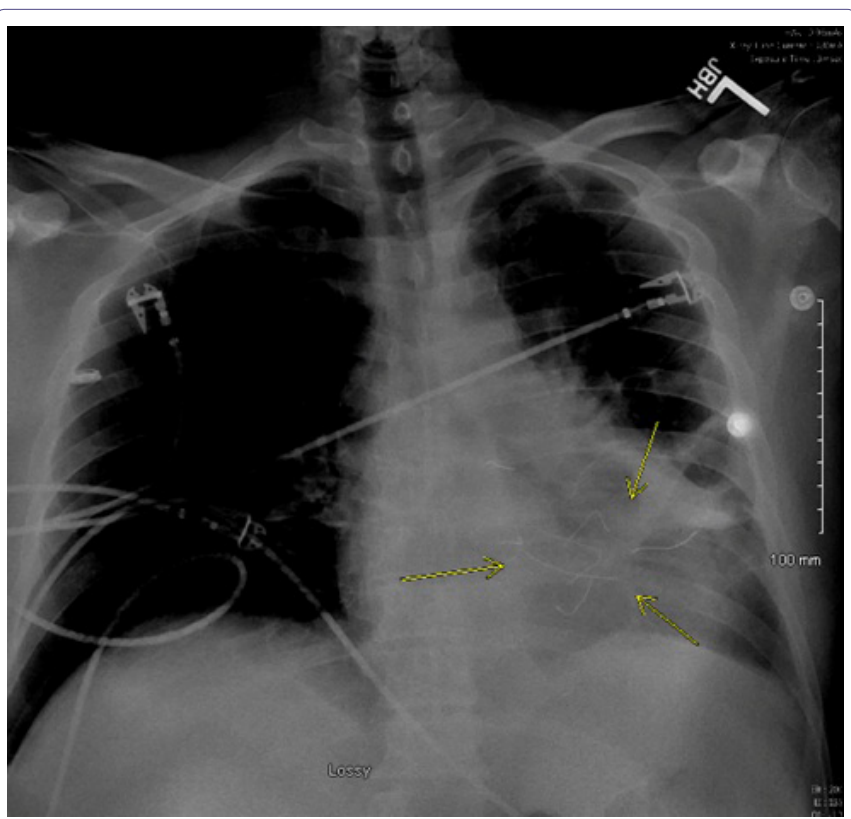

Figure 1: Chest x-ray showing copper wires.

direct response to a voice commanding him to insert the wires into his heart to protect him from evil spirits. He explained that he thought the wires would ward off any negativity in his environment.

The medical management of this case is ongoing, and will involve careful extraction of the wires, two of which have penetrated his cardiac muscle. His injuries resulted in a pair of small ventricular septal defects which will need to be repaired. As for his mental status, the patient verbalized understanding of how his self-imposed medication holiday exacerbated his psychosis. He was counseled on the danger of skipping doses of clozapine, and he agreed that it is in his best interest to remain dedicated to managing his disease.

\section{Discussion}

Self-mutilation is entirely too common in patients suffering from schizophrenia or schizoaffective disorder, along with bipolar disorder and mood disorders [3]. In many cases, the injuries sustained can be prevented by adherence to prescribed medical treatment regimens. There are various reports of non-suicidal self-mutilation in the literature, and it is first relevant to delineate between true suicide attempts and non-suicidal gestures when evaluating the significance of this case. For example, one particular case describes the insertion of a hot poker into a patient's chest [4]. This case, however, was an act that was performed with the intention of ending the patient's life. Considering that our patient expressly denied suicidal ideation, we focused on other cases that were not acts of suicide, but were simply acts of self-mutilation. Reports of non-suicidal gestures largely detail acts such as genital mutilation or self-enucleation of the eyes. Raslan et al., [5] published a case of penile self-mutilation in the context of psychopathology. Ciorba et al., [6] performed an analysis of facial self-mutilation, reporting the prevalence in populations with psychiatric disorders to be as high as 20\%. Jones [7], Harish et al., [8], and Gaur et al., [9] have all described cases in which patients inflicted injuries to their own eyes in acts of self-mutilation, while Reichstein et al., [10] describes a case of attempted self-enucleation by two psychotic incarcerated individuals. Mork et al., [11] looked closer at all types of non-suicidal self-harm in patients with schizophrenia, and performed a cross-sectional study of this population. Their results showed that $30 \%$ of their schizophrenia cohort had attempted some form of self-harm without suicidal intent at least one time. Among this population, the median number of attempts was six. The most common method of self-injury was cutting of the skin, and the act had been planned for less than an hour in $65 \%$ of cases.

The topic of intrathoracic foreign body insertion was reviewed by Jamilla et al., [12] in the context of a case report of pneumothorax resultant from needle insertion into the myocardium. In the presented case, no prior psychiatric history was known. The authors also discuss 8 other cases of reported intracardiac needles, 7 of which were self-inflicted. None of these references indicates needle insertion in response to command hallucinations.

It is evident that the medical burden of self-injurious behavior can be severe. In most cases, the risk of self-injury can be dramatically reduced with adherence to medication regimens. The importance of adherence to clozapine is especially vital, as this medication carries a severe risk of withdrawal psychosis [13], which likely played a large part in our presented case.

\section{References}

1. Challis S, Nielssen O, Harris A, Large M (2013) Systematic meta-analysis of the risk factors for deliberate self-harm before and after treatment for first-episode psychosis. Acta Psychiatri Scand 127: 442-454.

2. Malaspina D, Owen MJ, Heckers S, Tandon R, Bustillo J, et al. (2013) Schizoaffective Disorder in the DSM-5. Schizophr Res 150: 21-25.

3. Haddock G, Eisner E, Davies G, Coupe N, Barrowclough C (2013) Psychotic symptoms, self-harm and violence in individuals with schizophrenia and substance abuse problems. Shizophrenia Research 151: 215-220.

4. Roy AD (1967) True Heartburn. British Medical Journal 4: 279-280.

5. Raslan M, Donaldson J, Royle J (2015) Penile self-harm: a case report and concise clinical review. Scand Journal of Urol 3: 1-3.

6. Ciorba I, Farcus O, Giger R, Nisa L (2014) Facial self-mutilation: an analysis of published cases. Postgrad Med Journal 90: 191-200.

7. Jones NP (1990) Self-enucleation and psychosis. Br J Ophthalmol 74: 571573.

8. Harish T, Chawan N, Rajkumar RP, Chaturvedi SK (2012) Bilateral self-enucleation in acute transient psychotic disorder: the influence of sociocultural factors on psychopathology. Compr Psychiatry 53: 576-578.

9. Gaur S, Bist HK, Sinha V, Gupta M (2013) An unusual case of self-inflicted multiple needles injuries to eye. Indian J of Ophthalmol 61: 516-517.

10. Reichstein D, Esmaili N, Wells T, Kim JE (2015) Attempted auto-enucleation in two incarcerated young men with psychosis. Saudi $\mathrm{J}$ of Ophthalmol 29: 172-174.

11. Mork E, Walby FA, Harkavy-Friedman JM, Barrett EA, Steen NE, et al. (2013) Clinical characteristics in schizophrenia patients with or without suicide attempts and non-suicidal self-harm--a cross-sectional study. BMC Psychiatry 13: 255.

12. Jamilla FP, Casey LC (1998) Self-inflicted intramyocardial injury with a sewing needle: a rare cause of pneumothorax. Chest 113: 531-534.

13. Miller R (2009) Mechanisms of action of antipsychotic drugs of different classes, refractoriness to therapeutic effects of classical neuroleptics, and individual variation in sensitivity to their actions: Part II. Curr Neuropharmacol 7: $315-330$ 